\title{
Diversity of high risk human papilloma viruses in women treated with antiretroviral and in healthy controls and discordance with cervical dysplasia in the South of Benin
}

Callinice D. Capo-chichi ${ }^{*}$, Blanche Aguida ${ }^{1}$, Nicodème W. Chabi ${ }^{1}$, Jocelyn Acapko-Ezin ${ }^{2}$, Jonas Sossah-Hiffo ${ }^{1}$, Vidéhouénou K. Agossou ${ }^{2}$, Toussain Anagbla ${ }^{3}$, Marcel Zannou², Fabien Houngbé ${ }^{2}$ and Ambaliou Sanni ${ }^{1}$

\begin{abstract}
Background: High risk oncologic Human Papilloma Virus (HPV) is one of the leading causes of cervical cancer worldwide. We investigated HPV genotypes among women living or not with Human Immuno-deficiency Virus (HIV) in two major hospitals in the south of the republic of BENIN in the city of Cotonou. Our objective is to investigate the association of high risk-HPV to cervical dysplasia among women under stringent anti-retroviral (ARV) treatment and in controls without HIV.

Methods: The investigation was carried out within 1 year period in two groups of adult women: one group with HIV1 infection and under ARV therapy in the National University Hospital (CNHU-HKM) designated as CH group $(n=86)$; and one control group without HIV infection and attending the hospital Mènontin for routine gynecologic checkup and designated as ME group $(n=86)$. Cells derived from cervical uterine smears (CUS) were used for this investigation. The samples in ME group were selected to have similar lamin A/C profile with $\mathrm{CH}$ group. HPV genotypes were assessed by polymerase chain reaction (PCR) while lamin A/C expression profile was assessed by western blotting to corroborate the risk of cervical dysplasia.

Results: HPV56 is dominant in CH group while HPV66 is dominant in ME group. 31 \% of women in CH group are infected with HPV compared to $23 \%$ in ME group. Quadruple and quintuple HPV infections are more observed among $\mathrm{CH}$ group but not in ME group making HPV counts of 43 in $\mathrm{CH}$ group and 27 in ME group. Cervical dysplasia are present in $5 \%(4 / 86)$ of women in $\mathrm{CH}$ group and in $1 \%(1 / 86)$ of women in ME group at the time of CUS collection. The adjustment of the risk to develop cervical cancer in the future related to HPV infection and the total loss of lamin A/C is not significantly different in both groups.
\end{abstract}

Conclusion: Women living with HIV are more sensitive to multiple HPV infection but not all HPV infections generated cervical dysplasia. The effectiveness of antiretroviral therapy in $\mathrm{CH}$ group may reduce significantly the frequency of cervical dysplasia.

Keywords: HIV, ARV, HPV, lamin A/C deficiency, Cervical dysplasia

\footnotetext{
* Correspondence: callinice.capochichi@gmail.com

${ }^{1}$ Molecular Biomarkers in Cancer and Nutrition (BMCN), Unit of Biochemistry

and Molecular Biology (UBBM), Institute of Biomedical Sciences and

Applications (ISBA), Faculty of Sciences and Technologies (FAST), University

Abomey-Calavi (UAC), 04BP488, Cotonou, Benin

Full list of author information is available at the end of the article
} 


\section{Background}

Human Papilloma Viruses (HPV) viruses come in diverse genotypes. High risk HPVs (HR-HPV) are some of the leading causes of cervical cancer worldwide [1-6]. Over 200 genotypes have been identified and the HRHPVs are linked to the occurrence of cervical carcinomas [1-6]. Health disparities in developed and developing countries render some population such as African, Asian, Hispanic and Mexican women more vulnerable to these diseases [6]. The co-infection HPV/HIV is more investigated due to the collaborative effects of both viruses to generate cervical dysplasia $[7,8]$. HPV infections are more observed in population living with HIV due to the deregulation of the immune system $[9,10]$. Underneath the complex interaction between nuclear components and viral components toward cancer development, the stability of lamin $\mathrm{A} / \mathrm{C}$ should always be taken into consideration $[11,12]$. Although the high frequency of cervical dysplasia among women living with HIV was often associated to the co-infection HPV/HIV [1-8]; there are factors else than HPV that may also be involved in cervical dysplasia [11, 12]. Studies performed in four West African countries including Benin, has shown that cervical cancer ranks number one in women living with HIV and number second in women negative for HIV [13]. The present study was done to verify the HPV genotypes among women living with HIV and treated with ARV compared to women with no HIV infection and the association to the loss of lamin A/C and cervical dysplasia in the city of Cotonou (South of the Republic of BENIN).

\section{Methods}

\section{Population study}

The investigation was carried out within 1 year period in two groups of adult women: one group with HIV1 infection and under ARV therapy in the National University Hospital (CNHU-HKM), designated as $\mathrm{CH}$ group ( $n=$ 86); and one control group without HIV infection and attending the hospital Mènontin for gynecologic routine checkup and designated as ME group $(n=86)$. Women in group $\mathrm{CH}$ with HIV1 infection are under stringent anti-retroviral treatment at CNHU-HKM while women in group $\mathrm{ME}$ without $\mathrm{HIV}$ infection are not receiving any treatment. The $\mathrm{CH}$ group is under free stringent ARV treatment program offered by the Ministry of Health in BENIN.

The inclusion criteria are women of 20 to 60 years old (average 38 years) and sexually active. The exclusion criteria are women in menstruation or within third month post-partum. Signed Informed consent was obtained from all women before the collection of cervical uterine smears (CUS). Sample collections were carried out in the gynecological service under the supervision of a gynecologist and nurses. The Cervical Uterine junction was visualized with colposcopy before CUS collection $[11,12]$. This study was approved by the Research Ethic Committee of the Institute of Biomedical Science and Applications (CER-ISBA), and by the Ministry of Health in Republic of BENIN. HIV status was determined in the referral hospital (CNHU-HKM) with a rapid HIV test (Determine ${ }^{(\mathrm{R})}$, Abbott Diagnostics) or Genie $2^{(\mathrm{R})}$ (Bio-Rad, Marnes-La-Coquette, France) test as previously reported [13].

\section{Cell collection and processing}

To collect CUS for HPV and lamin A/C analyses, a disposable sterile speculum was introduced in the vagina along with a disposable cytobrush to reach the cervicaluterine junction where cells were collected by rotating the cytobrush clockwise twice. The brush was placed in a $50 \mathrm{ml}$ collection tube containing $5 \mathrm{ml}$ of sterile ice cold phosphate buffer saline (PBS) to collect cells. Cells were kept on ice and delivered within an hour to the laboratory (Unit of Biochemistry and Molecular Biology UBBM, Cotonou, BENIN) for processing. All collection tubes were centrifuged to gather cell pellets which were washed once with ice cold PBS before splitting them in several Eppendorf tubes (i) for the extraction of genomic DNA to genotype HR-HPV; (ii) for the analysis of lamin A/C proteins by western blot. The procedures followed were in accordance with the ethical standards of our institutional committee on human experimentation and in accordance with the Helsinki Declaration as previously reported [12].

\section{Reagents}

HPV multiplex primers, Agarose powder, Ethylenediaminetetraacetic acid (EDTA), 2- deoxyribonucleic acid (DNA) ladder and ethidium bromide were from Sigma-Aldrich (France). Tris-Base, glycine, sodium dodecyl sulfate, bis-acrylamide and nitrocellulose membrane, the protein ladder and the peroxidase conjugated secondary antibodies anti-rabbit and antimouse were purchased from Bio-Rad Inc (USA). Sodium chloride $(\mathrm{NaCl})$, potassium chloride $(\mathrm{KCl})$, Tween-20, protease inhibitor phenyl-methyl-sulfonyl fluoride (PMSF), 2-mercaptoethanol, methanol, glycerol, 1,4-Dithiothreitol (DTT), sodium fluoride $(\mathrm{NaF})$, sodium azide $\left(\mathrm{NaN}_{3}\right)$, Tris-Hydrochloride (tris$\mathrm{HCl}$ ) and sodium dodecyl sulfate (SDS, $\mathrm{NaC}_{12} \mathrm{H}_{25} \mathrm{SO}_{4}$ ) were from Sigma-Aldrich (USA). The primary antibody against lamin $\mathrm{A} / \mathrm{C}$ was purchased from Transduction Lab (USA). Antibody against ß-tubulin was from Santa Cruz Biotechnology (CA, USA). The chemo-luminescence reagent "Super Signal West Dura Extended Duration Substrate" made by PIERCE was from Thermo Scientific (Rockford, IL USA) and was used on Western blot membranes for protein revelation after exposure to X-ray films [12]. 


\section{DNA extraction}

DNA was extracted from cell pellets (i) by the phenolchloroform method after cell membrane disruption with lysis buffer containing proteinase $\mathrm{K}(20 \mathrm{mg} / \mathrm{ml})$ and RNAse as previously reported [12]. Phenol was added to the cell lysate $(\mathrm{V} / \mathrm{V})$ and centrifuged at $10,000 \mathrm{rpm}$ at $4{ }^{\circ} \mathrm{C}$ for $10 \mathrm{~min}$ to collect the upper aqueous phase into a new Eppendorf tube followed by the addition of chloroform $(\mathrm{V} / \mathrm{V})$ and another centrifugation $\left(10,000 \mathrm{rpm}\right.$ at $4{ }^{\circ} \mathrm{C}$ for $10 \mathrm{~min})$. The supernatant was collected in a new Eppendorf tube and ice cold ethanol (96\%) was added at $-20{ }^{\circ} \mathrm{C}$ for $4 \mathrm{~h}$ to precipitate DNA. The DNA pellet was recovered after centrifugation $\left(12,000 \mathrm{rpm}\right.$ at $4{ }^{\circ} \mathrm{C}$ for $\left.10 \mathrm{~min}\right)$, was washed with ice cold ethanol (70 \%) and then collected after centrifugation at $12,000 \mathrm{rpm}$ at $4{ }^{\circ} \mathrm{C}$ for $5 \mathrm{~min}$. The DNA pellet was air dried at $55^{\circ} \mathrm{C}$ for $20 \mathrm{~min}$, and solubilized in tris-EDTA (TE) buffer. The concentration of DNA was measured with a spectrophotometer $(260 \mathrm{~nm})$. Soluble DNA extract is stored in freezer until needed for genotyping of HPV by Polymerase chain reaction "PCR" [12].

\section{PCR and genotyping of HPV}

The quality of DNA was assessed by amplifying B-actin gene before setting up the PCR with HPV multiplex primers for genotyping. A nested multiplex PCR (NMPCR) assay that combines degenerate E6/E7 consensus primers and type specific primers was done as previously reported [12]. For each PCR reaction, positive and negative controls were used. A first consensus PCR was performed with human sequences (GPEG from SIGMA-Aldrich) to confirm the presence of HPV DNA. After the first PCR the amplified product was used again for a second amplification with the set of multiplex primers (nested PCR) to determine the genotype of HPV as previously described [12]. The multiplex primers used can detect the high grade oncogenic HPV and the low grade oncogenic HPV. The genotypes were divided in 4 groups according to the set of primers. The set 1 of primers is able to detect HPV16; HPV 18; HPV 31; HPV 59; HPV 45 and yield PCR products of 457; 322; 263; 215 and 151 base pairs (bp), respectively. The set 2 of primers is able to detect HPV 33; HPV 6/11; HPV 58; HPV 52; HPV 56; and yield PCR products of 398; 333; 274; 229 and 181 bp respectively. The set 3 of primers is able to detect HPV35; HPV 42; HPV 43; HPV 44 with PCR products of 358; 277; 219 and163 bp respectively. The set 4 of primers is able to detect HPV68; HPV 39; HPV 51; HPV 66; yielding PCR products of 333; 280; 223 and $172 \mathrm{bp}$ respectively. The PCR products were run through $1.5 \%$ agarose gel to separate amplicons according to their size. Amplified bands were detected by ethidium bromide staining under UV light. The HPV genotype was determined according to the molecular size of the band as specified previously [12].

\section{Cell lysates and lamin A/C analysis}

Cell pellets (ii) were put in lysis buffer A [ $50 \mathrm{mM}$ tris- $\mathrm{HCl}$ (pH 7.9), $150 \mathrm{mM} \mathrm{NaCl}, 0.1 \mathrm{mM}$ EDTA, $1 \% \mathrm{NP}-40$, $0.5 \mathrm{mM}$ DTT, $0.5 \mathrm{mM}$ PMSF, $30 \mathrm{mM} \mathrm{NaF}$ and $0.5 \%$ protease inhibitor cocktail] and kept on ice for $30 \mathrm{~min}$ with vigorous agitation every $5 \mathrm{~min}$ as previously described [12]. Samples were boiled in SDS sample buffer for $5 \mathrm{~min}$ and store in freezer until analysis. Before western blotting protein samples were boiled again, loaded on $7.5 \%$ SDSpolyacrylamide gels and run at 100 volts for $2 \mathrm{~h}$ in trisglycine buffer, followed by a transfer to nitrocellulose membranes with transfer buffer containing tris-glycine and $20 \%$ methanol. The membranes were blocked with $5 \%$ milk in $1 \mathrm{X}$ Tris-buffered-saline (TBS) containing $0.1 \%$ Tween-20 (TBST) for $30 \mathrm{~min}$ at room temperature before incubation in primary antibody rabbit-anti-lamin $\mathrm{A} / \mathrm{C}$ at room temperature. The blots were washed 4 times for $10 \mathrm{~min}$ with TBST and incubated with HRPconjugated secondary antibody anti-rabbit. The blots were washed 4 times for 15 min with TBST and incubated for 3 min in Super Signal West Dura Extended Duration Substrate before exposure to $\mathrm{x}$-ray film and a film developer for the detection lamin $\mathrm{A} / \mathrm{C}$ or of $\mathrm{B}$-tubulin as a loading control [12].

\section{Statistical analyses}

This study is a transversal case control study between HIV infected group $(\mathrm{CH})$ and non HIV group (ME). The Fisher test (F-test) was used on Excel software to compare the prevalence of HPV infection between groups $\mathrm{CH}$ and ME. The CUS with HPV are given the value of 0 if there are not infected with HR-HPV, the value of 1 if they are infected with one type of oncogenic HPV, the value of 2 if they are co-infected with two types of HR-HPV, the value of 3 if they are co-infected with three types of HR-HPV, the value of 4 when are co-infected with 4 types of HRHPV and the value of 5 when they are co-infected with five types of HR- HPV; the difference between group $\mathrm{CH}$ and $\mathrm{ME}$ is considered significant when $p<0.05$.

The non-parametric Mann-Whitney test was used to compare lamin A/C profile between both groups. Lamin $\mathrm{A} / \mathrm{C}$ profiles were given the value of 3 when normal expression was observed, the value of 2 for weak expression of lamin $\mathrm{A} / \mathrm{C}$ and the value of 0 for total absence of lamin $\mathrm{A} / \mathrm{C}$. The difference between group $\mathrm{CH}$ and $\mathrm{ME}$ groups is considered significant when $p<0.05$.

\section{Video colposcopy}

Women uterine cervix was examined with a video Colposcopy (SONY) by a gynecologist. Staining with dilute acetic 
acid followed by Lugol's iodine solution was done to reveal the cervical lesions if present. The lesions of the cervix appeared white when stained with acetic acid (visual inspection with acetic acid, VIAA+) and yellow when stained with Lugol's iodine solution (visual inspection with Lugol's solution, VILI) as previously reported $[11,12]$.

\section{Ethical considerations}

The protocol of this study including objectives, participants, sample collection, analysis, and reporting of data was approved by the Ethics Committee of the Institute of Biomedical Sciences and Applications (CER-ISBA) and by the Ministry of Health in Republic of BENIN. The objectives of the study were explained to all participants before inclusion. Signed informed consent was freely obtained from all participants before sample collections for the study [12].

\section{Results}

\section{HR-HPV status in HIV population (CH group)}

Dual, quadruple and quintuple HR-HPV type coinfections were observed in 7/86 (8\%) women in $\mathrm{CH}$ group, (HPV56, 58); (HPV58, 43); (HPV58, 42); (HPV 35, 42); (HPV 52, 42, 66, 39); (HPV 52, 43, 66, 39); (HP V56, 33, 42, 39, 68)\}. The HPV56 $(9 / 86=10 \%)$ is more frequent than HPV58 $(6 / 86=7 \%)$ and HPV42 $(6 / 86=$ $7 \%)$ followed by HPV $52(4 / 86=5 \%)$, HPV43(4/86 = $5 \%)$ and HPV $39(3 / 86=3 \%)$, HPV $45(2 / 86=2 \%)$, HPV 66 (2/86 = $2 \%)$, HPV35 (1/86 = $1 \%)$, HPV 33(1/86 $=1 \%)$ and HPV $31(1 / 86=1 \%)$ as shown in Fig. 1 . Unpredictably, HPV 18 and HPV16 were not detected in $\mathrm{CH}$ group. Overall, the co-infection $\mathrm{HR}-\mathrm{HPV} / \mathrm{HIV}$ is present in $31 \%$ of women in $\mathrm{CH}$ group while HPV counts including multiple HPV infections were 43.

\section{HR-HPV status in non-HIV population (group ME)}

Dual and triple HR-HPV co-infections were observed in 5/86 (5 \%) of women in group ME: [HPV 45, 56]; [HPV43, 44]; [HPV18, 66]; [HPV42, 59, 68]; [HPV42, 59, 39]. In group ME, HPV $66(4 / 86=5 \%)$ is more frequent than HPV58 (3/86 = $3 \%)$, HPV56 (3/86=3 \%), HPV33 $(3 / 86=3 \%)$ followed by HPV59 $(2 / 86=2 \%)$, HPV18 $(2 /$ $86=2 \%)$ and HPV45 (1/86=1 \%), HPV16 (1/86=1\%) as shown in Fig. 2. Overall, HR-HPV infection was observed in $23 \%$ women of ME group while total HRHPV counts including multiple HR-HPV infections were 27. The diversity of HR-HPV genotypes makes hard to use HPV as biomarker to follow women toward cancer development in both groups.

Analysis of HR-HPV prevalence in HIV and non-HIV populations (group $\mathrm{CH}$ vs group $\mathrm{ME}$ )

More women in $\mathrm{CH}$ group have significantly higher $\mathrm{HR}$ HPV infections $(27 / 86=31 \%)$ than women in ME group $(20 / 86=23 \%) ; p<0.001$, as shows in Fig. 3 and Table 1. HR-HPV counts is higher in $\mathrm{CH}$ group with HIV infections $(n=43)$ compared to ME group without HIV ( $n=$ 27), $p<0.001$. The length of ARV treatment in $\mathrm{CH}$ group varies between 1 to 15 years (Table 1 ).

\section{Cervical dysplasia in the context of HIV, HR-HPV and lamin A/C deficiency}

The prevalence of cervical dysplasia in both groups is significantly lower than the prevalence of HR-HPV

$\mathrm{HPV}$ incidence in $\mathrm{CH}$ group

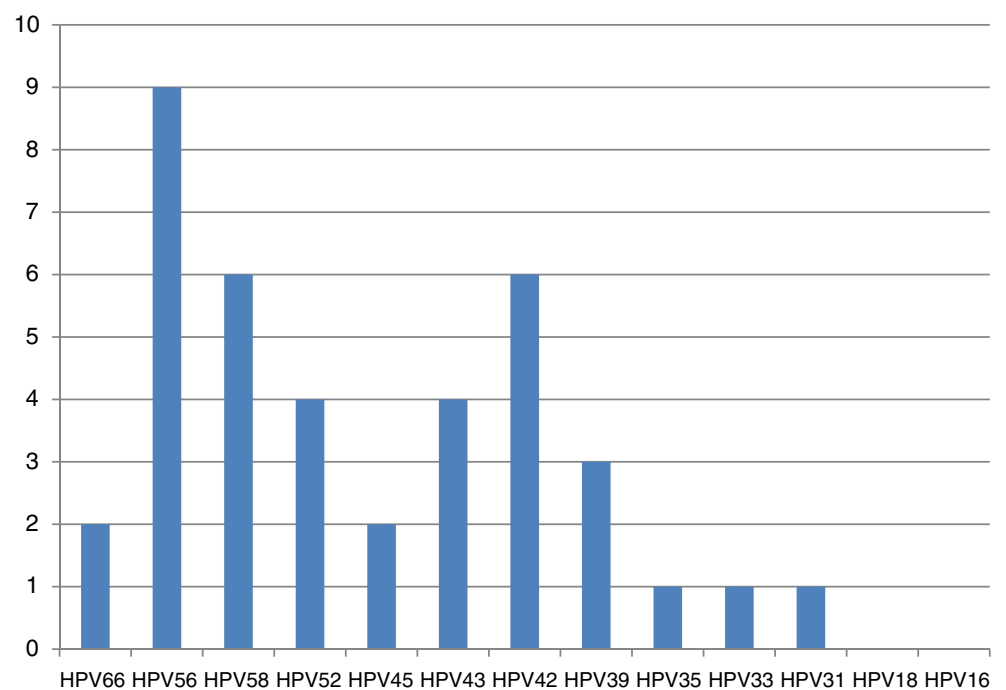

Fig. 1 Incidence of HPV types in HIV infected women in group CH. Genotype HPV56 is more frequent than HPV 58, HPV 42 followed by HPV52, HPV43; HPV56, HPV39; HPV45, HPV66 and HPV35, HPV33, HPV31. HPV18 and HPV16 were not detected in this CH group 


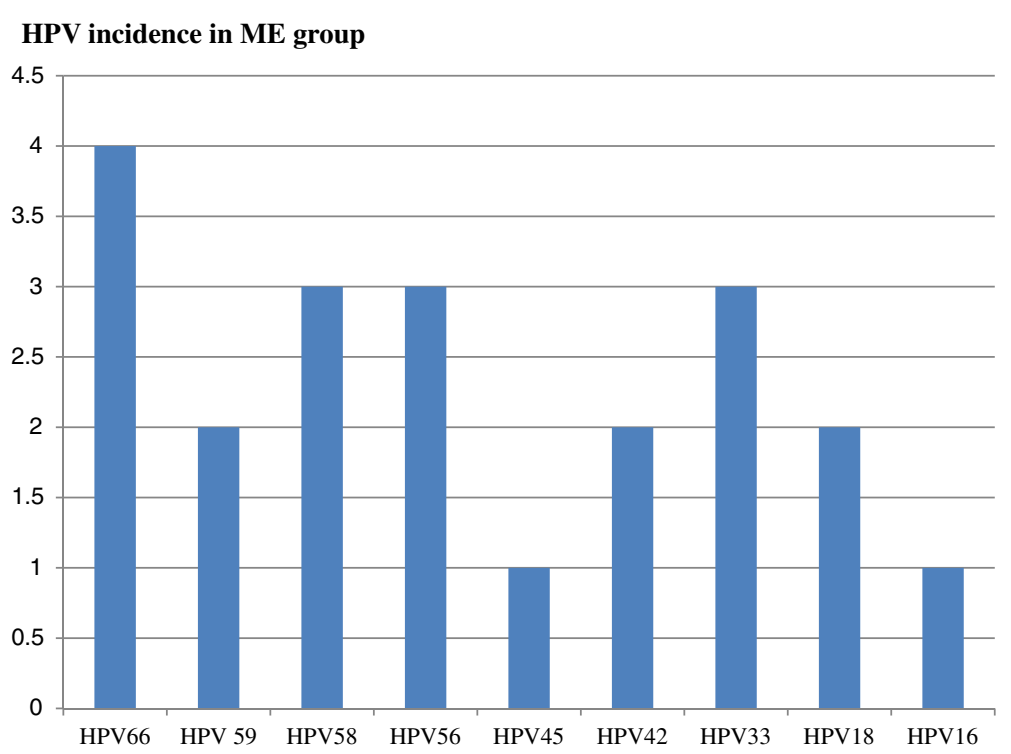

Fig. 2 Incidence of HPV type in women without HIV infection in group ME. Genotype HPV 66 is more frequent than HPV58, HPV56, HPV33 followed by HPV59, HPV18 and HPV45, HPV16

infections and it is opportune in this instance to evaluate the risk to develop cervical dysplasia with the deficiency in lamin A/C. An example of western blot performed with cell lysates derived from CUS samples in $\mathrm{CH}$ group is shown in Fig. 4 with samples expressing lamin $\mathrm{A} / \mathrm{C}$ and without lamin A/C. Sample A1 expresses lamin A/C while samples $\mathrm{C} 1$ and $\mathrm{C} 2$ have totally lost the expression of lamin A/C. Samples C1 and C2 have more risk to develop cervical cancer regardless of HIV and HR-HPV status. Multiple HR-HPV co-infections were most observed in women with low lamin $\mathrm{A} / \mathrm{C}$ or no lamin $\mathrm{A} / \mathrm{C}$ along with cervical lesions (Figs. 5 and 6).

In $\mathrm{CH}$ group cervical dysplasia was observed in 4/86 (5\%) of women living with HIV. We observed low grade cervical intraepithelial lesions $(\mathrm{CIN}-1)$ in one woman (52 years old) with co-infections HIV/HPV58 and HPV43; high grade lesions (CIN-2) in one woman (49 years old) with co-infections HIV/HPV52, HPV43,

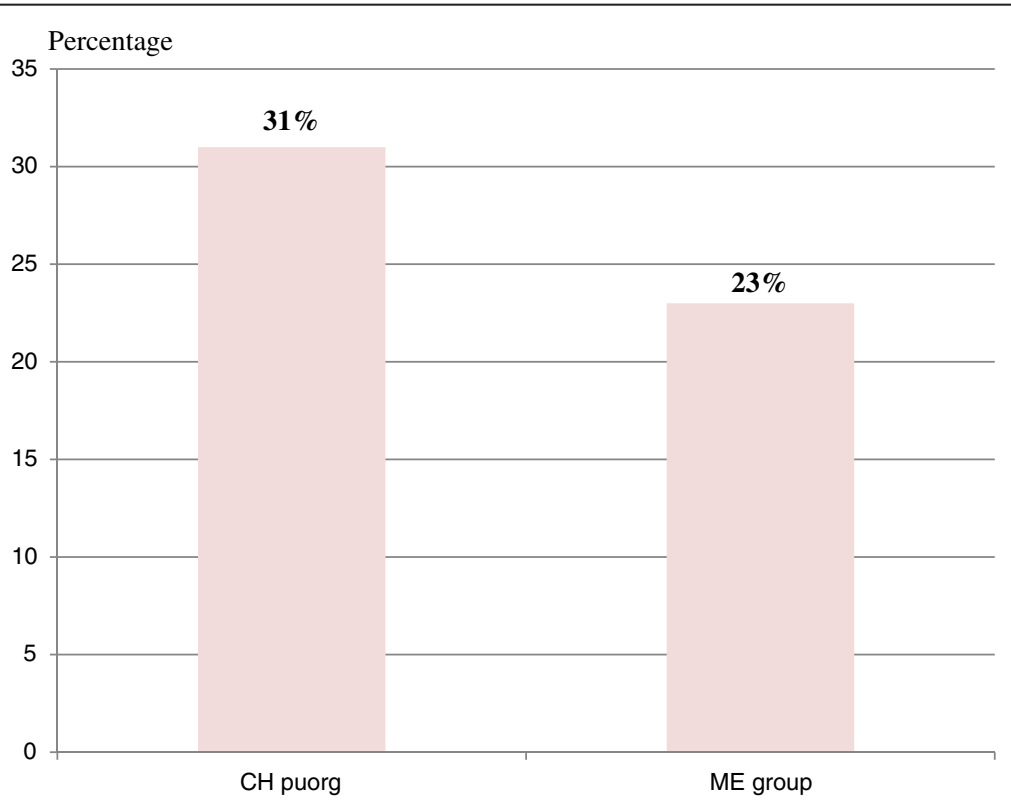

Fig. 3 Histogram representing the frequency of women infected with HPV in $\mathrm{CH}$ group with HIV and ME group without HIV. The percentage of women with HPV infection in CH group (31\%) is significantly higher than in ME group without (23\%) with $p<0.001$ 
Table 1 Summary of ARV treatment, lamin A/C, HPV and cervical lesions in CH and ME groups

\begin{tabular}{|c|c|c|c|c|c|c|c|}
\hline \multicolumn{4}{|l|}{$\mathrm{CH}$ group } & \multicolumn{4}{|l|}{ ME group } \\
\hline Lamin $\mathrm{A} / \mathrm{C}(n=86)$ & Women with HPVs & Cervical lesions & Years with ARV (y) & $\operatorname{Lamin} \mathrm{A} / \mathrm{C}$ & Women with HPVs & Cervical lesions & Years with ARV \\
\hline Normal $N=34$ & $9(10 \%)$ & 0 & $1-13$ years & Normal $N=35$ & $5(6 \%)$ & 0 & No \\
\hline Low $N=22$ & $7(8 \%)$ & 1 & $1-9$ years & Low $N=21$ & $3(3 \%)$ & 0 & No \\
\hline Absent $N=30$ & $11(13 \%)$ & 3 & $1-15$ years & absent $N=30$ & $12(14 \%)$ & 1 & No \\
\hline Total $N=86$ & $31 \%$ & 5 & $1-15$ years & $N=86$ & $23 \%$ & 1 & No \\
\hline
\end{tabular}

Table representing the frequency of HPV infection in antiretroviral (ARV) treated women (CH group) and non HIV infected control (ME group). The HPV frequency does not correlate with cervical lesions

HPV 66 and HPV39; condylomatous epithelial lesions in one woman (26 years old) with co-infections HIV/ HPV56, HPV33, HPV42, HPV39 and HPV68; condylomatous epithelial lesions in one woman (28 years old) with co-infections HIV/HPV HPV35 and HPV42. One woman with co-infections HIV/HPV52, HPV42, HPV66 and HPV39 has not developed cervical lesions at the time of CUS collection as shown in Fig. 5. The silencing of lamin $\mathrm{A} / \mathrm{C}$ is observed in $34 \%$ of group $\mathrm{CH}$ and this put them at risk to develop cervical dysplasia in the future. All 4 women with cervical lesions in $\mathrm{CH}$ group have lost the expression of lamin $\mathrm{A} / \mathrm{C}$ at the time of CUS collections associated with co-infection $\mathrm{HIV} / \mathrm{mul}-$ tiple HR-HPVs. Conversely not all HIV/multiple HRHPV co-infections developed cervical lesions at the time of sample collection. The histogram integrating lamin $\mathrm{A} / \mathrm{C}, \mathrm{HR}-\mathrm{HPV}$ and cervical lesions data in $\mathrm{CH}$ group infected with HIV is shown in Fig. 5 and verified that cervical lesions are more associated to the deficiency of lamin $\mathrm{A} / \mathrm{C}$ than to HR-HPV infection.

In ME group without HIV infection, one woman with HPV18 has developed cervical dysplasia (CIN I) at the time of sample collection (1/86 or $1 \%$ ) associated to the loss of lamin A/C. All other women with HPV infection

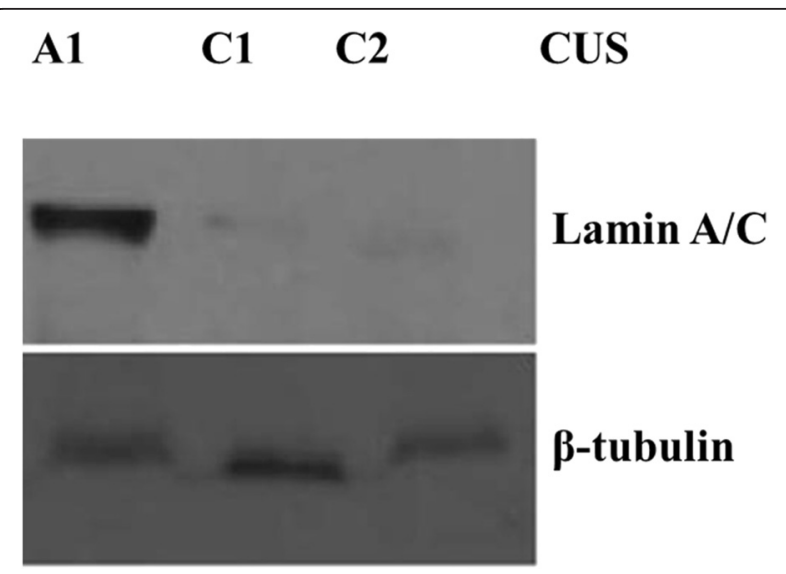

Fig. 4 Silencing of Lamin $A / C$ in cervical uterine smears (CUS). An example of western blot showing lamin A/C in CUS samples of women in $\mathrm{CH}$ group. Sample A1 expresses of lamin A/C while samples $C 1$ and $C 2$ have lost the expression of lamin $A / C$ have not developed cervical lesions at the time of sample collection but the silencing of lamin $\mathrm{A} / \mathrm{C}$ is observed in $34 \%$ of them and this put them at risk to develop cervical dysplasia in the future (Fig. 6). Table 1 represents the prevalence of HR-HPV infection among antiretroviral (ARV) treated women ( $\mathrm{CH}$ group) and non HIV infected control (ME group) and shows that HR-HPV infection is not always associated with cervical lesions.

When considering the subgroup with lamin A/C deficiency, the prevalence of $\mathrm{HR}-\mathrm{HPV}$ infection in $\mathrm{CH}$ group is $13 \%$ while the prevalence of HR-HPV infection in ME group is $14 \%$ as shown in table; the difference was not significant. In both groups the absence of lamin $\mathrm{A} / \mathrm{C}$ is a suitable prognostic factor for the risk to develop cervical cancer in the future.

\section{Two years follow-up of the women participating in this study}

Both groups are under the supervision of gynecologists and are advised to perform CUS or colposcopy once a year. We recalled all women investigated after our first analysis in Mai 2014 for a 2 years follow-up in June 2016. Among the $\mathrm{CH}$ group, no new cases of cervical dysplasia were reported; unexpectedly the woman with co-infections HIV/HPV52, HPV42, HPV66 and HPV39 with no cervical lesions at the time of CUS collection did not develop cervical lesions 2 years later but did clear out all HPV genotypes as shown by gel photo in Fig. 7 (CUS FCU-177) along with negative control (CUS FCU-176). The 26 years old woman with quintuple HRHPV (HPV56, HPV33, HPV42, HPV39 and HPV68) and condylomatous lesions is still receiving ARV treatment and is in good health. All other women are still receiving ARV treatment for free and are in good health. Among the ME group, one woman with HPV and no lamin A/C developed cervical lesions and two women with no HPV but with total absence of lamin A/C developed cervical lesions and were treated by the gynecologist. Sample numbers are scrambled and could not be traced to the patients.

\section{Discussion}

HR-HPV genotypes fluctuate in each country and environment as well as the association of HIV/HR-HPV co- 


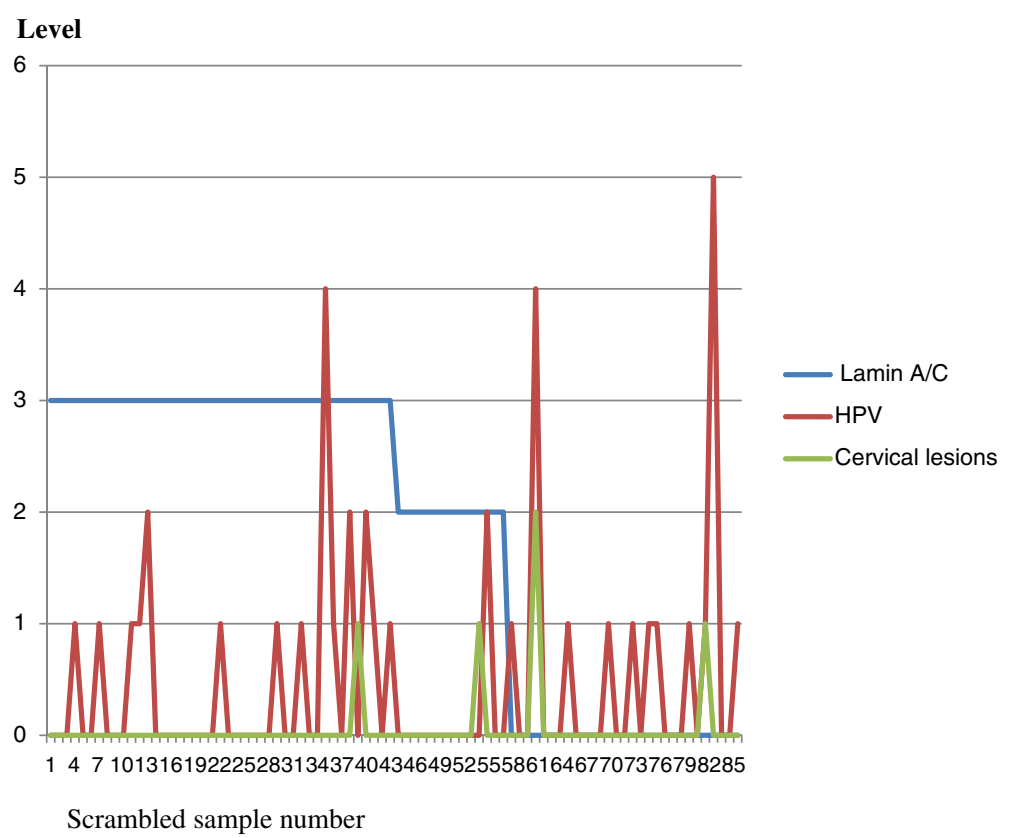

Fig. 5 Histogram integrating HPV, lamin A/C and cervical lesions data in $\mathrm{CH}$ group infected with HIV. In this $\mathrm{CH}$ group cervical lesions are more associated to low or absence of lamin A/C than it is associated to HPV infection

infections to cervical dysplasia or cervical cancer $[1,3,12]$. Our study in the south of BENIN shows that HPV56 is predominant among women leaving with HIV and is more frequent than HPV 58 and HPV42 and HPV 66. Among women in control ME group with no HIV infection, the
HPV 66 is predominant and is more frequent than HPV58, HPV56.

In study done in Democratic Republic of the Congo (Kinshasa) the most common HR-HPV types among HIV-positive women were HPV68, HPV35, HPV52 and

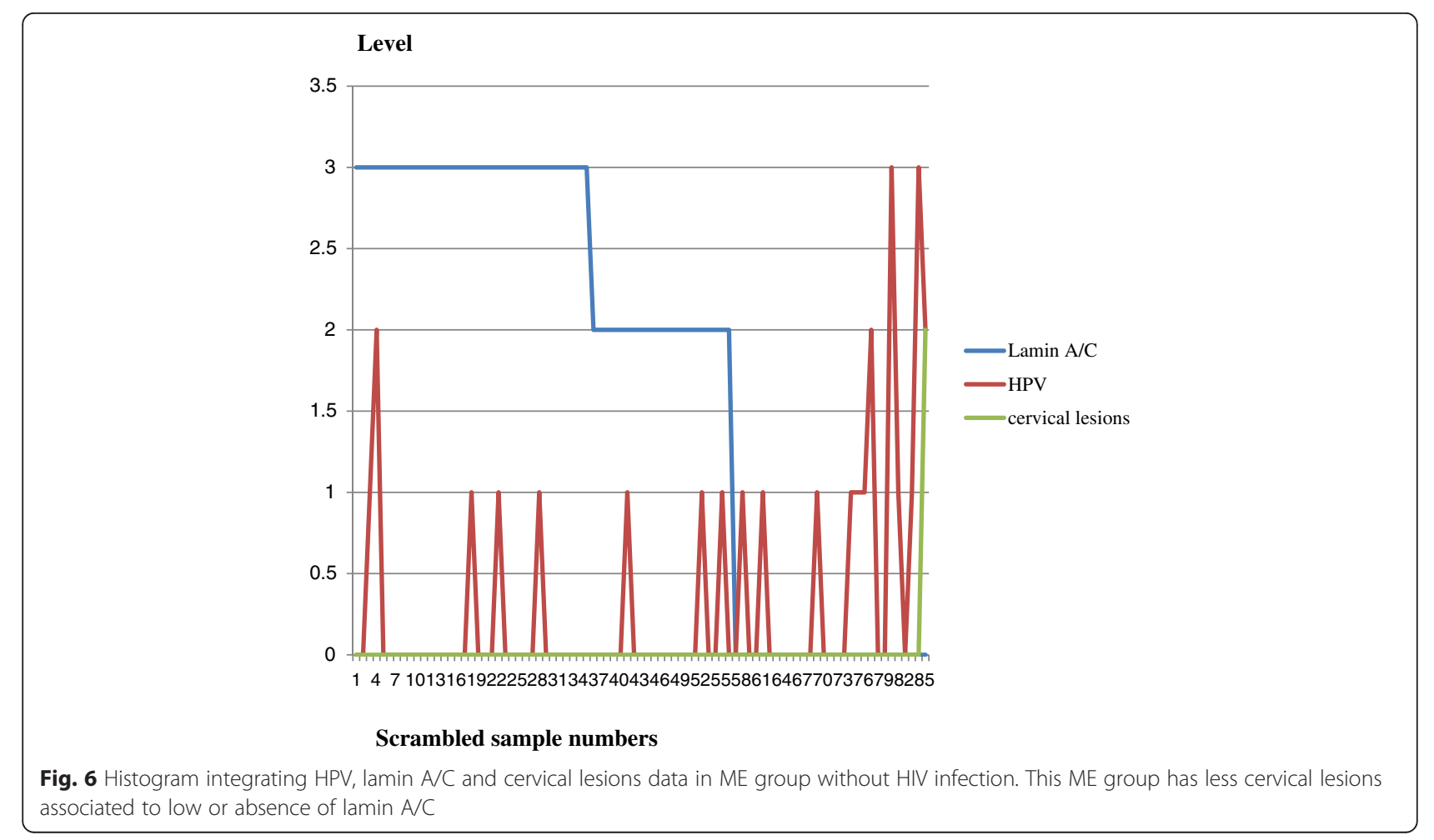




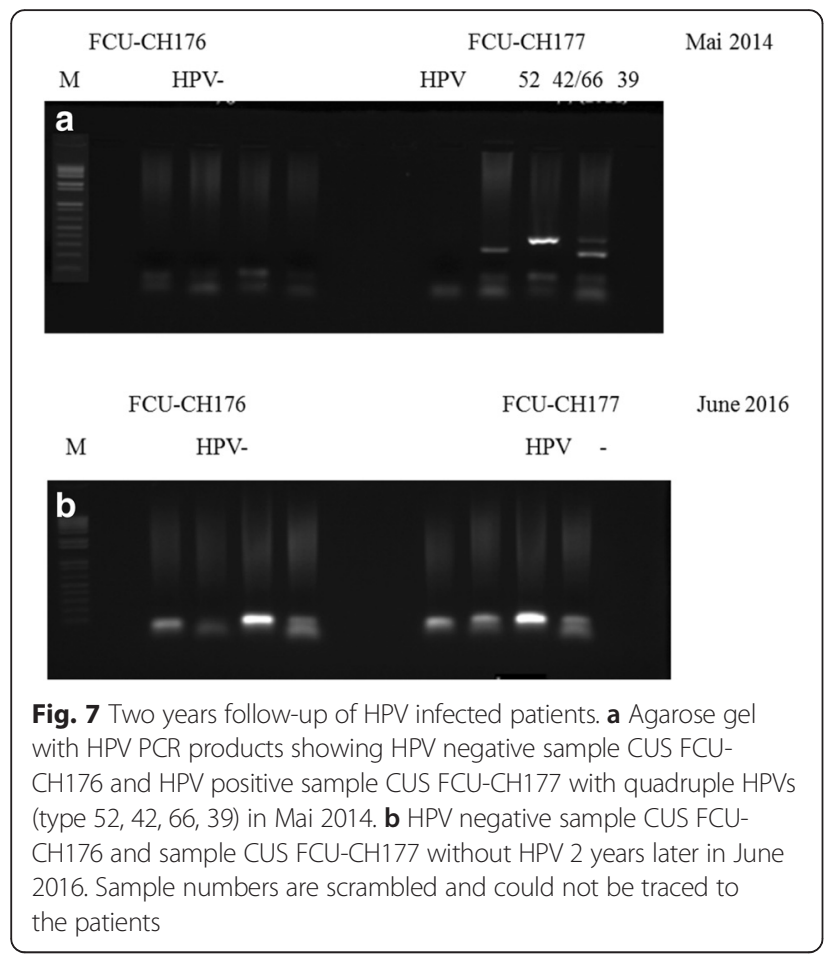

HPV16. Among women with negative/unknown HIV status, the most common HR-HPV types were HPV52, HPV35 and HPV18 as reported [1]. In southwest China (Yunnan) the most common HR-HPV types in women infected with HIV were HPV52, HPV58, HPV18, HPV16, and HPV33 as reported [4]. In Thailand the most common HR-HPV types in the population were HPV72, HPV52, HPV62, and HPV16 [3].

Although HR-HPV infections are more frequent in $\mathrm{CH}$ group with HIV (Fig. 4), not all women infected with HR-HPV will develop cervical dysplasia or cancer (Figs. 5 and 6) and it is opportune to integrate other cancer biomarkers with HR-HPV analyses to efficiently predict the risk to develop cervical dysplasia independently to viral infection. The discrepancy between the low rate of cervical intra epithelial lesions and the high rate of HR$\mathrm{HPV}$ infections is striking in $\mathrm{CH}$ group and is an indicator that the evaluation of HR-HPV genotypes solely is not sufficient to define the risk to develop cervical lesions in women living with HIV and under efficient ARV treatment. The early detection of cervical lesions in women with HIV allowed the gynecologist to apply cryotherapy for these women.

Aside of viral integration into the genome, nuclear protein anomalies and chromosomal instabilities occur to initiate tumor formation and progression [14-17]. The deficiency of lamin A/C was already demonstrated in several studies to be a reliable prognostic factor for cervical, ovarian, breast, prostate, gastro-intestinal, colon cancers and neuroblastoma [11, 12, 14-21]. In BENIN, cervical cancer ranked number one among women infected with HIV but appreciative to the efficacy of ARV treatments received in $\mathrm{CH}$ group the prevalence of cervical dysplasia is not enormous as predicted by studies based on HR-HPV infection rate [8, 13]. HPV did clear out from some women without triggering cervical lesions as shown reported by our 2-year follow-up periods.

Although the prevalence of HPV infection is significantly higher in the $\mathrm{CH}$ group, the frequency of $\mathrm{HR}$ HPV infection adjusted to the deficiency of lamin $\mathrm{A} / \mathrm{C}$ is not significantly different in both groups investigated making this assessment more predictable of the risk to develop cervical cancer in the future. Due to the diversity of virus types involved in cervical cancer development and progression, we recommend to investigate further more molecular biomarkers in living individual for the screening and prevention of cervical cancer rather than taking solely into consideration HR-HPV infection rate especially in African countries.

\section{Conclusion}

Women living with HIV are more sensitive to multiple HR-HPV infections but not all HR-HPV infections generate cervical dysplasia. The effectiveness of antiretroviral therapy in $\mathrm{CH}$ group may reduce significantly the frequency of cervical dysplasia although it has no influence on the prevalence of HR-HPV/HIV co-infection. Lamin A/C should always be used as biomarker for cervical cancer screening associated to HR-HPV genotypes in African countries to not skip women who develop cervical dysplasia independently of HR-HPV infections.

\section{Abbreviations}

ARV, Anti-retroviral; CUS, cervical uterine smears; EDTA, Ethylenediaminetetraacetic acid (EDTA); HIV, Human immunodeficiency Virus; HPV, Human Papilloma Virus; HR-HPV, high risk Human Papilloma Virus; HRP, Horseradish Peroxidase; $\mathrm{KCl}$, potassium chloride; $\mathrm{NaCl}$, Sodium chloride; $\mathrm{NaF}$, sodium fluoride; $\mathrm{NaN}_{3}$, Sodium azide; PBS, phosphate Buffered Saline; PMSF, PhenylMethyl-Sulfonyl Fluoride; SDS, sodium dodecyl sulfate; TBS, Tris Buffer Saline; TBST, Tris Buffer Saline plus Tween-20; Tris-HCl, Tris-Hydrochloride

\section{Acknowledgments \\ We thank Dr. Georges Offrin MD; Nurse WANOU Sylvia and their colleagues in the Gynecology/obstetric service, Hospital Mènontin as well as Nurse AGBODJELOU BOURAIMA Latifatou and her colleagues in the Gynecology/ obstetric service, of National University Hospital (CNHU-HKM) for their efforts in CUS sample collections. We thank Pr. LALEYE Anatole, director of ISBA for allowing us to use his cytogenetics lab equipment. We thank the radiology Crew of CNHU-HKM for their help throughout our research investigation. We address special thanks to Pr. SINSIN A. Brice, Rector of the University Abomey-Calavi (UAC) in BENIN and all the staff members of UAC for their constant encouragement and support for all our innovative projects towards cancer research in Benin. We also thank our colleagues (Dr. Xu Xiang-Xi, Smith R. Elizabeth and Moore Robert) of Ovarian Cancer Program at the Sylvester Cancer Center, Miller Medical School at the University of Miami, Florida, USA for their collaboration and support.}

\section{Funding}

We do not have actual funding but this research was supported by collaboration efforts between researchers of the Unit of Biochemistry and 
Molecular Biology (UBBM) of the University Abomey Calavi, Medical doctors of the National University Hospital (CNHU-HKM) and Mènontin Hospital in BENIN.

\section{Authors' contributions}

$\mathrm{CDC}: \mathrm{PhD}, \mathrm{MPH}$ : is the principal investigator of this project and she has designed and organized this study and has trained PhD students to perform western blot and PCR for this study. BA, PhD. Student: has performed sample collection, western blotting and HPV genotyping. NWC, PhD: has provided reagents and technical support and advices for HPV genotyping. JAE, MD: is one of the responsible in charge of the National Program to treat HIV patients with ARV in CNHU-HKM, Cotonou. JSH: PhD. Student: has HPV genotyping. VKA, MD: is one of the Gynecologists who performed

Colposcopic cervical-uterine examination and diagnosis of cervical dysplasia. TA: is one of the Gynecologist who performed Colposcopic cervical-uterine examination and diagnosis of cervical dysplasia. MZ, MD: is chief of the National Program to treat HIV patients in CNHU-HKM, Cotonou, BENIN. FH, MD: is chief of the National Program to treat cancer patients in CNHU-HKM Cotonou, BENIN. AS, PhD: is the director of the UBBM and responsible of HPV detection program at the Institute of Biomedical Sciences and Applications (ISBA) in BENIN; he provided technical support and advices for HPV genotyping. All authors read and approved the final manuscript.

\section{Competing interests}

The authors declare that they have no competing interests.

\section{Consent for publication}

Not applicable.

\section{Ethics approval and consent to participate}

The protocol of this study is approved by the Ethics Committee of the Institute of Biomedical Sciences and Applications (CER-ISBA) and by the Ministry of Health in Republic of BENIN. Signed informed consent was obtained freely from all participants before sample collections for the study.

\section{Author details}

${ }^{1}$ Molecular Biomarkers in Cancer and Nutrition (BMCN), Unit of Biochemistry and Molecular Biology (UBBM), Institute of Biomedical Sciences and Applications (ISBA), Faculty of Sciences and Technologies (FAST), University Abomey-Calavi (UAC), 04BP488, Cotonou, Benin. ${ }^{2}$ National University Hospital (CNHU-HKM), Cotonou, Benin. ${ }^{3}$ Hospital Mènontin, BP 160 Cotonou, Benin.

Received: 22 April 2016 Accepted: 28 June 2016

Published online: 15 August 2016

\section{References}

1. Ali-Risasi C, Verdonck K, Padalko E, Vanden Broeck D, Praet M. Prevalence and risk factors for cancer of the uterine cervix among women living in Kinshasa, the Democratic Republic of the Congo: a cross-sectional study. Infect Agent Cancer. 2015;15(10):20.

2. da Silva L, Miranda A, Batalha R, Ferreira L, Santos M, Talhari S. High-risk human papillomavirus and cervical lesions among women living with HIV/ AIDS in Brazilian Amazon. Braz J Infect Dis. 2015;19:557-62.

3. Marks MA, Gupta S, Liaw KL, Tadesse A, Kim E, Phongnarisorn C, Wootipoom V, Yuenyao P, Vipupinyo C, Rugpao S, Sriplienchan S, Gravitt PE, Celentano DD. Prevalence and correlates of HPV among women attending family-planning clinics in Thailand. BMC Infect Dis. 2015;15:159.

4. Zhang $H Y$, Fei $M D$, Jiang $Y$, Fei $Q Y$, Qian $H$, Xu L, Jin YN, Jiang CQ, Li $H X$, Tiggelaar SM, Smith JS, Sahasrabuddhe W, Qiao YL. The diversity of human papillomavirus infection among human immunodeficiency virus-infected women in Yunnan. China Virol J. 2014;11:202-3.

5. Scarinci IC, Garcia FA, Kobetz E, Partridge EE, Brandt HM, Bell MC, Dignan M, Ma GX, Daye JL, Castle PE. Cervical Cancer Prevention: New Tools and Old Barriers. Cancer. 2010;116:2531-42.

6. Madeddu G, Mameli G, Capobianco G, Babudieri S, Maida I, Bagella P, Rocca G, Cherchi PL, Sechi LA, Zanetti S, Nunnari G, Dessole S, Mura MS. HPV infection in HIV-positive females: the need for cervical cancer screening including HPV-DNA detection despite successful HAART. Eur Rev Med Pharmacol Sci. 2014;18:1277-85.

7. Adler DH, Wallace M, Bennie T, Abar B, Meiring TL, Williamson AL, Bekker LG. Cumulative Impact of HIV and Multiple Concurrent Human
Papillomavirus Infections on the Risk of Cervical Dysplasia. Adv Virol; 2016:7310894

8. Jaquet A, Horo A, Ekouevi DK, Toure B, Coffie PA, Effi B, Lenaud S, Messou E, Minga A, Sasco AJ, Dabis F, leDEA West Africa Collaboration. Risk Factors for Cervical Intraepithelial Neoplasia in HIV Infected Women on Antiretroviral Treatment in Côte d'Ivoire, West Africa. PLoS One. 2014;9:e90625.

9. Martins AE, Lucena-Silva N, Garcia RG, Welkovic S, Barboza A, Menezes ML, Maruza M, Tenório T, Ximenes RA. Prevalence of human papillomavirus infection, distribution of viral types and risk factors in cervical samples from human immunodeficiency virus-positive women attending three human immunodeficiency virus-acquired immune deficiency syndrome reference centres in northeastern Brazil. Mem Inst Oswaldo Cruz. 2014;109:738-47.

10. McKenzie ND, Kobetz EN, Ganjei-Azar P, Rosa-Cunha I, Potter JE, Morishita A, Lucci JA 3rd, Guettouche T, Hnatyszyn JH, Koru-Sengul. HPV in HIV-infected women: implications for primary prevention. Front Oncol. 2014;4:179.

11. Capo-Chichi CD, Aguida B, Cai QK, Offrin G, Agossou KV K, Sanni A, Xu XX. The Deficiency of Nuclear Proteins GATA6 and Lamin A/C as Prognostic Factor for Cervical Neoplasia. American Journal of Cancer Prevention. 2015:3:109-16.

12. Capo-Chichi CD, Aguida B, Chabi NW, Cai QK, Offrin G, Agossou VK, Sanni A, $\mathrm{Xu} X X$. The Deficiency of Nuclear Proteins lamin $A / C$ is an independent risk factor for cervical cancer. Cell Oncol (Dordr). 2016;39:59-68.

13. Jaquet A, Odutola M, Ekouevi DK, Tanon A, Oga E, Akakpo J, Charurat M, Zannou MD, Eholie SP, Sasco AJ, Bissagnene E, Adebamowo C, Dabis F. leDEA Cancer and HIV infection in referral hospitals from four West African countries. West Africa Collaboration Cancer Epidemiol. 2015:39:1060-5.

14. Capo-chichi CD, Cai KQ, Simpkins F, Ganjei-Azar P, Godwin AK, Xu XX. Nuclear envelope structural defects cause chromosomal numerical instability and aneuploidy in ovarian cancer. BMC Med. 2011;9:28.

15. Capo-chichi CD, Cai KQ, Smedberg J, Ganjei-Azar P, Godwin AK, Xu XX. Loss of A-type lamin expression compromises nuclear envelope integrity in breast cancer. Chin J Cancer. 2011;30:415-25.

16. Matsumoto A, Hieda M, Yokoyama Y, Nishioka Y, Yoshidome K, Tsujimoto M, Matsuura N. Global loss of a nuclear lamina component, lamin A/C, and LINC complex components SUN1, SUN2, and nesprin-2 in breast cancer. Cancer Med. 2015;4:1547-57.

17. Saarinen I, Mirtti T, Seikkula H, Boström PJ, Taimen P. Differential Predictive Roles of A- and B-Type Nuclear Lamins in Prostate Cancer Progression. PLoS One. 2015;10:e0140671.

18. Gong G, Chen P, Li L, Tan H, Zhou J, Zhou Y, Yang X, Wu X. Loss of lamin A but not lamin $C$ expression in epithelial ovarian cancer cells is associated with metastasis and poor prognosis. Pathol Res Pract. 2014;211:175-82.

19. Wang AS, Kozlov SV, Stewart CL, Horn HF. Tissue specific loss of A-type lamins in the gastrointestinal epithelium can enhance polyp size. Differentiation. 2015;89:11-21.

20. Belt EJ, Fijneman RJ, van den Berg EG, Bril H, Delis-van Diemen PM, Tijssen $M$, van Essen HF, de Lange-de Klerk ES, Beliën JA, Stockmann HB, Meijer S, Meijer GA. Loss of lamin A/C expression in stage II and III colon cancer is associated with disease recurrence. Eur J Cancer. 2011;47:1837-45.

21. Nardella M, Guglielmi L, Musa C, lannetti I, Maresca G, Amendola D, Porru M, Carico E, Sessa G, Camerlingo R, Dominici C, Megiorni F, Milan M, Bearzi C, Rizzi R, Pirozzi G, Leonetti C, Bucci B, Mercanti D, Felsani A, D'Agnano I. Down-regulation of the Lamin $A / C$ in neuroblastoma triggers the expansion of tumor initiating cells. Oncotarget. 2015;6:32821-40.

\section{Submit your next manuscript to BioMed Central and we will help you at every step:}

- We accept pre-submission inquiries

- Our selector tool helps you to find the most relevant journal

- We provide round the clock customer support

- Convenient online submission

- Thorough peer review

- Inclusion in PubMed and all major indexing services

- Maximum visibility for your research

Submit your manuscript at www.biomedcentral.com/submit 